\title{
Second harmonic spectroscopy of semiconductor nanostructures
}

Østergaard, John Erland; Yu, Ping; Bozhevolnyi, Sergey I.; Hvam, Jørn Märcher; Ledentsov, N. N.

\section{Published in:}

Proceedings of QELS'99

Link to article, DOI:

10.1109/QELS.1999.807595

Publication date:

1999

Document Version

Publisher's PDF, also known as Version of record

Link back to DTU Orbit

Citation (APA):

Østergaard, J. E., Yu, P., Bozhevolnyi, S. I., Hvam, J. M., \& Ledentsov, N. N. (1999). Second harmonic spectroscopy of semiconductor nanostructures. In Proceedings of QELS'99 (pp. 233-234). Optical Society of America. https://doi.org/10.1109/QELS.1999.807595

\section{General rights}

Copyright and moral rights for the publications made accessible in the public portal are retained by the authors and/or other copyright owners and it is a condition of accessing publications that users recognise and abide by the legal requirements associated with these rights.

- Users may download and print one copy of any publication from the public portal for the purpose of private study or research.

- You may not further distribute the material or use it for any profit-making activity or commercial gain

- You may freely distribute the URL identifying the publication in the public portal 


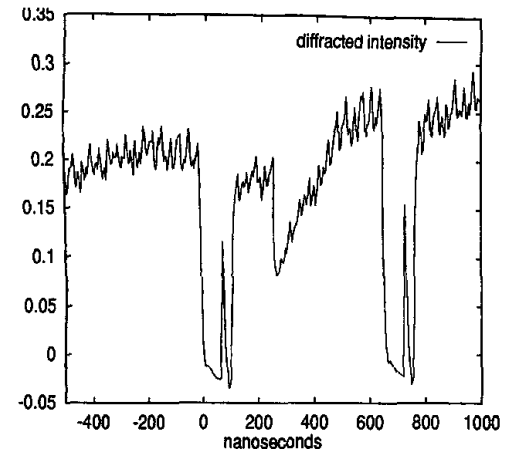

QThJ4 Fig. 1. Time-resolved diffraction on a nanosecond time-scale.

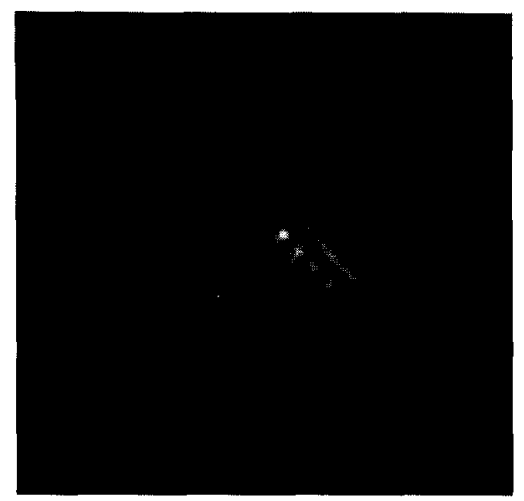

QThJ4 Fig. 2. 60 ps x-ray pulse with uv calibration pulses separated by 6 ps.

changes in the long range order of the semiconductor InSb. The experimental setup is at the Advanced Light Source synchrotron at Lawrence Berkeley National Laboratory. A 150 fs Ti:Sapphire laser system giving $2.5 \mathrm{~mJ} /$ pulse at a $1 \mathrm{kHz}$ repetition rate is phase-locked with -5 ps jitter to the electron bunches in the storage ring. The laser pulse, which initiates the dynamics, is overlapped spatially and temporally with the $\mathrm{x}$-rays on an InSb wafer with the (111) crystal planes cut 16 degrees from the surface. The use of asymmetric-cut crystals

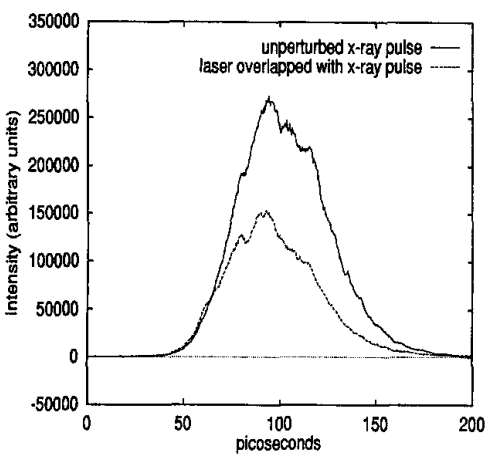

QThJ4 Fig. 3. Laser-induced changes within the $\mathrm{x}$-ray pulse. The $\mathrm{x}$-ray pulse is modified following the initiation of a structural change within the crystal by an ultrashort laser pulse. better matches the penetration depths of the laser and the $\mathrm{x}$-rays.

To investigate dynamics on a nanosecond time-scale, the synchrotron bunch structure is effectively a continuous source. Figure 1 shows the time-resolved diffraction efficiency of the crystal superimposed on the time-structure of the synchrotron, measured using an avalanche photo-diode. Off the peak of the rocking curve, three effects are evident: 1) A sudden decrease due to a loss of order in the crystalline lattice. 2) A complete recrystallization of a melted surface layer on a $100 \mathrm{~ns}$ timescale. 3) An increase due to lattice expansion which shifts the crystal onto the Bragg peak.

Since the dynamics are happening faster than the diode time-resolution, we have employed an X-ray streak camera with 3 ps resolution. ${ }^{3}$ We take advantage of the fact that the sample regrows following excitation, to average over many shots. The temporal jitter is reduced by using a photoconductive switch to trigger the sweep plates of the camera. In Fig. 2, we show a streak corresponding to a single $60 \mathrm{ps} x$-ray pulse from the synchrotron. Below the $x$-ray streak are images of $4 \mathrm{UV}$ pulses, each separated by 6 ps which calibrates the time axis.

After initiating a structural transition during the $x$-ray pulse, we then follow the loss of order in the material on a ps time-scale. Figure 3 shows an unperturbed $x$-ray pulse together with one in which the laser is incident on the sample, modifying the $\mathrm{x}$-ray pulse shape.

At the highest fluences below damage threshold, we observe evidence of a melting time within the streak camera temporal resolution. Further experiments and discussion of this point will be presented.

${ }^{*}$ Lawrence Berkeley National Laboratory, Berkeley, California 94720 USA; E-mail: paheimann@ lbl.gov

* Lawrence Livermore National Laboratory, Livermore, California 94551 USA; E-mail: missalla@ grace.lbl.gov

tUniversity of Michigan Department of Physics and Center for Ultrafast Optical Science, Ann Arbor, Michigan 48109 USA; E-mail: liao@ eecs.umich.edu

$¥$ Clarendon Laboratory, University of Oxford OX13PU, United Kingdom; E-mail: jsw@ermine.ox.ac.uk

1. V. Srajer et al., Science 274, 1726 (1996).

2. J. Larsson et al., Appl. Phys. A. 66, 587 (1998).

3. J. Larsson et al., Optics Letters 13, 1012 (1997).

\section{QThJ5}

3:30 pm

\section{Second harmonic spectroscopy of} semiconductor nanostructures

J. Erland, P. Yu, S.I. Bozhevolnyi, J.M. Hvam, N.N. Ledentsov, ${ }^{*}$ Mikroelektronik Centret, The Technical University of Denmark, Build. 345 E, DK-2800 Lyngby, Denmark; E-mail: jeo@mic.dtu.dk

Semiconductor nanostructures and their application to optoelectronic devices ${ }^{1}$ have attracted much attention recently. Lowerdimensional structures, and in particular

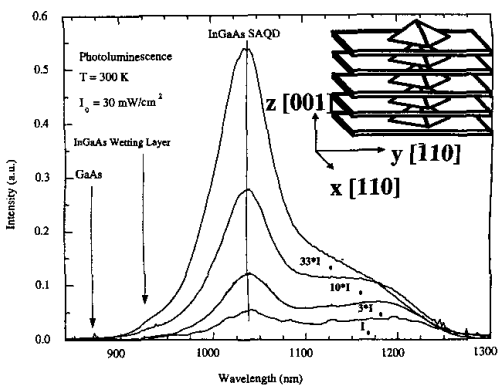

QThJ5 Fig. 1. The photoluminescence characterization at room temperature of our 20 layered InGaAs sample with GaAs spacer layers. The inset shows the structure.

quantum dots, are highly anisotropic resulting in broken symmetry as compared to their bulk counterparts. This is not only reflected in highly anisotropic linear polarization properties, as studied recently in pyramide-shaped self-assembled InGaAs quantum dots, ${ }^{2}$ but also in second harmonic generation (SHG), which can be greatly enhanced allowing for detailed studies of such structures. ${ }^{3}$ SHG has contributed considerably as a technique to investigate solid state systems where the local inversion symmetry is broken by e.g. a surface or an interface, defect states or simply by structures so small that the bulk symmetry properties no longer are valid. ${ }^{4}$ Our idea is to use SHG in the configurations, where the bulk and surface contributions are forbidden for a homogeneous sample, so that the only source of SHG is associated with nanostructures embedded in the host material. Our first measurements in GaAS showed that indeed the SHG in reflection at normal incidence from a (001)substrate, where SHG from bulk and surface is forbidden by symmetry, was more than 3 orders of magnitude weaker than that from a (111)-substrate. We can therefore study various nanostructures embedded in the $\mathrm{GaAs}$ host material. In this work we focus on SHG from an MBE-grown sample consisting of 20 $\mathrm{In}_{0.5} \mathrm{Ga}_{0.5}$ As layers spaced by $5 \mathrm{~nm}$ GaAs layers. The InGaAs layers are grown to a StranskiKrastranow phase transition resulting in the formation of pyramide-shaped self-assembled quantum dots with base of $18 \mathrm{~nm}$ and height of $5 \mathrm{~nm}$ laterally spaced by $55 \mathrm{~nm}$ on average on top of a wetting layer. ${ }^{2}$ The HeNe excited luminescence spectra at room temperature have the quantum dot states as the main spectral component. However, both the GaAs spacer layers and InGaAs wetting layers are observed as shoulders in the spectra, see Fig. 1. In Fig. 2 we show our first results of SHG from this type of semiconductor structure. At a fundamental wavelength of $900 \mathrm{~nm}$ the SHG signal is linearly dependent on the number of InGaAs layers that may be associated with the vertical coupling of quantum dots, ${ }^{2}$ see inset of Fig. 2. Moreover, measuring the SHG at different wavelengths we can identify both the GaAs spacer layers and the InGaAs wetting layers, see Fig. 2. Both layers are expected to have rather weak SHG generation, however, the formation of the quantum dots in between the two layers break the symmetry and gives SHG. The peak at $856 \mathrm{~nm}$ from the GaAs spacer layers are 


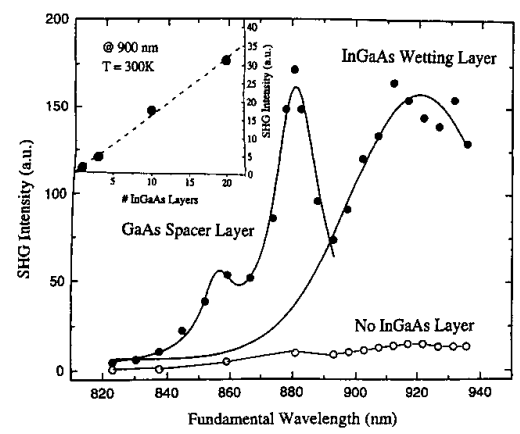

QThJ5 Fig. 2. The second harmonic generation signal versus wavelength showing the resonance from the GaAs spacer layer and the InGaAs layers with symmetries broken due to quantum dot formation. Compare to identical measurements in a GaAs sample with no InGaAs layers. Inset show the linear dependence of the SHG signal at $900 \mathrm{~nm}$ of the number of InGaAs layers.

attributed to the enhanced SHG from the thin quantum well, whereas the peak at $880 \mathrm{~nm}$ is attributed to the enhanced (due to the nanostructure) SHG from the GaAs substrate. The wider SHG resonance reflects the InGaAs wetting layers. These results show that indeed we can use SHG for spectroscopy of semiconductor nanostructures, as the SHG signal from a pure GaAs sample is very weak and unstructured as expected. Our current work focusses on measuring not only the quantum dot $\mathrm{SHG}$, but also further on the symmetry properties as they will be reflected in the SHG. Another avenue for us will be to use SHG to obtain high spatial resolution in the experiments ${ }^{5}$ and avoid spectral blurring from the inhomogeneous distribution of electronic states, that can be an obstacle for obtaining accurate information on these important nanostructures.

${ }^{*}$ The A.F. Ioffe Institute, St. Petersburg 194021, Russia

1. Heinrichsdorff et al., Appl. Phys. Lett. 71, 22 (1997).

2. Yu et al., submitted to Phys. Rev. B

3. Tomassini et al., Appl. Phys. Lett. 73, 2245 (1998).

4. T.F. Heinz, in: Nonlinear Surface Electromagnetic Phenomena, H. Ponath and G. Stegeman, eds., Elsevier, Amsterdam, 353 (1991).

5. Bozhevolnyi et al., Optics Comm. 150, 49 (1998). Lewis et al., Opt. Lett. 23, 1111 (1998)

\section{QThJ6}

3:45 pm

Optical properties of planar photonic band gap structures measured with femtosecond pulses

Joel Boyd, Peng Jiang, Kevin Huang, Jane Bertone, Vicki Colvin,

Daniel Mittleman, ${ }^{*}$ Department of Chemistry, MS-60, Rice University, 6100 Main St.

Houston, Texas 77005 USA

There has been a great deal of recent interest in systems which exhibit periodic dielectric properties. Over the last decade, numerous examples of such systems have been constructed, some with

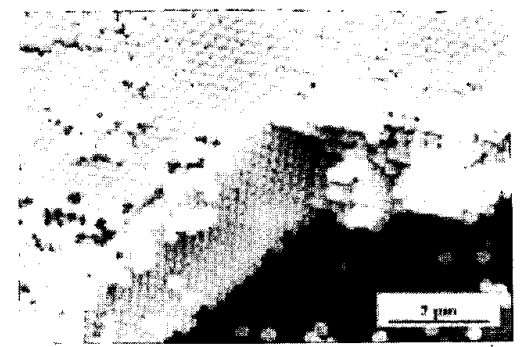

QThJ6 Fig. 1. SEM photograph of a photonic crystal of $330 \mathrm{~nm}$ diameter silica spheres, with $\sim 15$ layers. The [111] crystal axis is normal to the planar surface on which the crystal is grown.

rather complicated three-dimensional structure. Because of diffraction off of the periodic structure, such systems can exhibit a photonic band gap, a region of the spectrum where no propagating electromagnetic waves can exist. Structures with appropriate symmetries could exhibit a band gap at a particular frequency regardless of the angle of incidence of the radiation, i.e., a 3-dimensional band gap.

The early work in this area focused primarily on periodicity with length scales which were readily accessible to micromachining or other mesoscopic assembly techniques. ${ }^{1}$ As a result, these systems exhibit band gaps in the microwave or sub-millimeter range. More recently, attention has been turned to photonic band systems in the optical regime, which require sub-micron periodicity in the dielectric constant. ${ }^{2,3,4}$ Band gaps in the visible and nearinfrared have been achieved using lithographic etching of semiconductors as well as colloidal assembly of sub-micron dielectric spheres. For the most part, these samples are either quite thin, comprising at most a few layers of periodic dielectric variation, or extremely thick, as in the case of colloidal crystals grown in capillary tubes. In both cases, a quantitative comparison between theory and experiment is problematic, either because of the lack of systematic thickness control or because of the high crystal defect density in many of these examples.

Recently, we have fabricated photonic band gap structures, using ordered crystalline arrays of highly monodisperse silica spheres. ${ }^{5}$ These spheres, which exhibit only $\sim 3 \%$ variation in radius, can be induced to crystallize in closepacked arrays. These arrays can be evaporated onto any planar surface, for excellent compatibility with conventional optical coating technologies. The resulting systems are extremely well ordered with uninterrupted periodicity over at least $40 \mu \mathrm{m} \times 40 \mu \mathrm{m}$ area; they are virtually free of crystalline defects, and exhibit a periodic dielectric with an index contrast of $\mathrm{n}_{\text {silica }} / \mathrm{n}_{\text {air }} \sim 1.4$. This is a large enough contrast to exhibit substantial photonic band gap behavior, although not yet large enough for a full three-dimensional band gap. ${ }^{6}$ Because the diameter of the silica spheres can be chosen more or less arbitrarily, materials with optical stop bands at any wavelength in the visible and near-infrared can be fabricated. Most importantly, careful control of the evaporation rate provides good control of the thickness of these arrays, ranging from a few up to several hun- dred layers. Figure 1 shows an edge-view SEM image of one of these samples.

With these samples, it is now possible to perform quantitative comparisons with theoretical treatments of optical photonic band gap materials. In order to do so, it is desirable to measure not only the extinction coefficient $\alpha(\lambda)$ of the sample, but also the dispersion $\mathrm{n}(\lambda)$. Far from the photonic band gap, the dielectric constant of the sample is simply given by the average of the dielectric constants of air and silica, weighted by the volume fraction occupied by each. Near the band edge, however, the refractive index deviates dramatically from this volume-weighted average. In optical transmission measurements, this effect becomes increasingly difficult to measure as one approaches the band gap, because of the strong decrease in the transmitted light intensity. This difficulty is compounded by imperfections in the sample. A measurement of the full complex dielectric function of these samples would be an important test of the models for photonic band gap behavior. Recently, Watson and co-workers' have made such measurements by inserting a colloidal sample in one arm of a Mach-Zender interferometer. Using a cw tunable laser, they were able to map both $\alpha(\lambda)$ and $n(\lambda)$ over a wide range, and thus extract the full dielectric function $\varepsilon(\omega)$ of the periodic structure.

Here we propose an alternative method for measuring $\varepsilon(\omega)$. We fabricate the photonic structure directly on the reflecting surface of a conventional mirror. We then use this mirror as one end of a Michaelson interferometer, constructed for the purpose of performing in terferometric autocorrelations of femtosecond pulses. By comparing the interferometric autocorrelations measured with and without the sample in place, it should be possible to extract the optical constants of the sample. This method has the advantage that it is broadband; one obtains information on $\alpha(\lambda)$ and $n(\lambda)$ over the full bandwidth of the femtosecond pulse. The data can be acquired rapidly, without tuning or adjusting any optical elements other than translating the mirror on which the sample is grown. Moreover, it does not require any additional instrumentation, beyond that used in a conventional interferometric autocorrelator. One obvious disadvantage is that information can only be obtained within the tuning range of the mode-locked laser used in these measurements, roughly $750 \mathrm{~nm}-900 \mathrm{~nm}$. However, this is sufficient for detailed comparisons with theory.

In this preliminary measurement, we have used $\sim 110$ femtosecond pulses from a selfmode-locked Ti:sapphire laser. The sample consists of $\sim 19$ layers of silica spheres, of $\sim 350 \mathrm{~nm}$ diameter, grown on a microscope slide which was first coated with silver to form a reflective coating. Figure 2 shows the optical density (measured in transmission) of a sample with similar characteristics. This sample/ mirror was placed in one arm of the interferometer, at the focus of an $\sim \mathrm{f} / 10$ lens to reduce the spot size on the sample somewhat. The interferometric autocorrelation is measured with a $150 \mu \mathrm{m}$ thick KDP crystal. Immediately after measuring the autocorrelation from the bare mirror, it is vertically translated 